\title{
POLARIZATION PROFILE NEAR THE 4430 BAND
}

\author{
K. NANDY and H. SEDDON
}

Royal Observatory, Edinburgh, U.K.

It has been reported by several authors that the extinction profile near the wavelengths of unidentified diffuse features shows fine structure (Brück and Nandy, 1970; Walker, 1971 ; York, 1971; Bromage, 1972); the question arises whether these features also exhibit fine structure in wavelength dependence of polarisation. It was first suggested by Greenberg et al. $(1970,1971)$ that interstellar bands produced by grain impurities may be polarized differently from the neighbouring continuum. Similar conclusions were also derived by Wickramasinghe and Nandy (1971) and Kelly (1971). Our first attempt to derive a profile of polarization against wavelength across the $4430 \AA$ band was reported earlier (Nandy and Seddon, 1970). In this method we have used a Wollaston prism at the Cassegrain focus of the $36^{\prime \prime}$ Telescope of the Royal Observatory, Edinburgh to produce orthogonally polarised spectra on the photographic plate. The results for the star 55 Cyg (Figure 1) indicated a definite profile. It was felt than an extension of the programme to acquire further profiles and to establish instrumental polarisation to a greater accuracy was necessary.

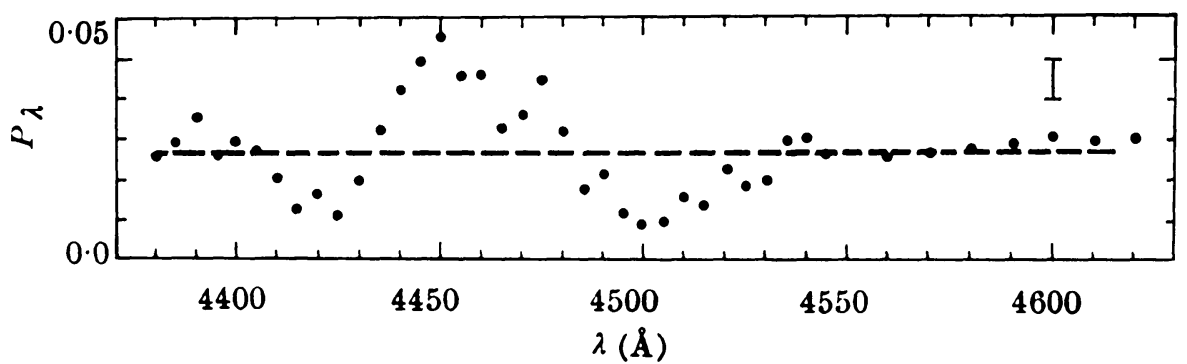

Fig. 1. Wavelength dependance of polarization across in $4430 \AA$ feature for 55 Cyg.

For partially plane polarized star light, the monochromatic magnitude difference $\Delta m_{\lambda}$ between the pair of orthogonally polarized spectra is given by

$$
\Delta m_{\lambda}(\alpha)=p_{\lambda} \cos 2\left(\alpha-\theta_{\lambda}\right),
$$

where

$p_{\lambda}=$ the amount of polarization expressed in magnitudes

$\alpha=$ the angle between the sky oriented reference axis and one of the two directions of the prism axis.

$0_{\lambda}=$ the position angle of the starlight measured from the same reference axis as $\alpha$.

The position of the Wollaston prism is adjusted so that initially $\alpha=0$. Stokes 
parameters $p_{x}(\lambda)$ and $p_{y}(\lambda)$ expressed in magnitudes are given by (see Hall and Serkowski, 1963)

$$
\begin{aligned}
& p_{x}(\hat{\lambda})=\Delta m_{\lambda}\left(0^{\circ}\right) \\
& p_{y}(\lambda)=\Delta m_{\lambda}\left(45^{\circ}\right) .
\end{aligned}
$$

Stokes parameters $p_{x}$ and $p_{y}$ have been determined for the unpolarized star $\alpha$ Cyg (Deneb) and for two other stars with differing degrees of polarization and $4430 \AA$ absorption. The spectra are measured and reduced digitally producing a complete plot of $p_{x}$ and $p_{y}$ against wavelength, as shown in Figure 2. The profiles derived from $\alpha$ Cyg establish the instrumental polarization profile, which must then be compared with the profiles of the other stars to ascertain the significance of the difference inside and outside the band.

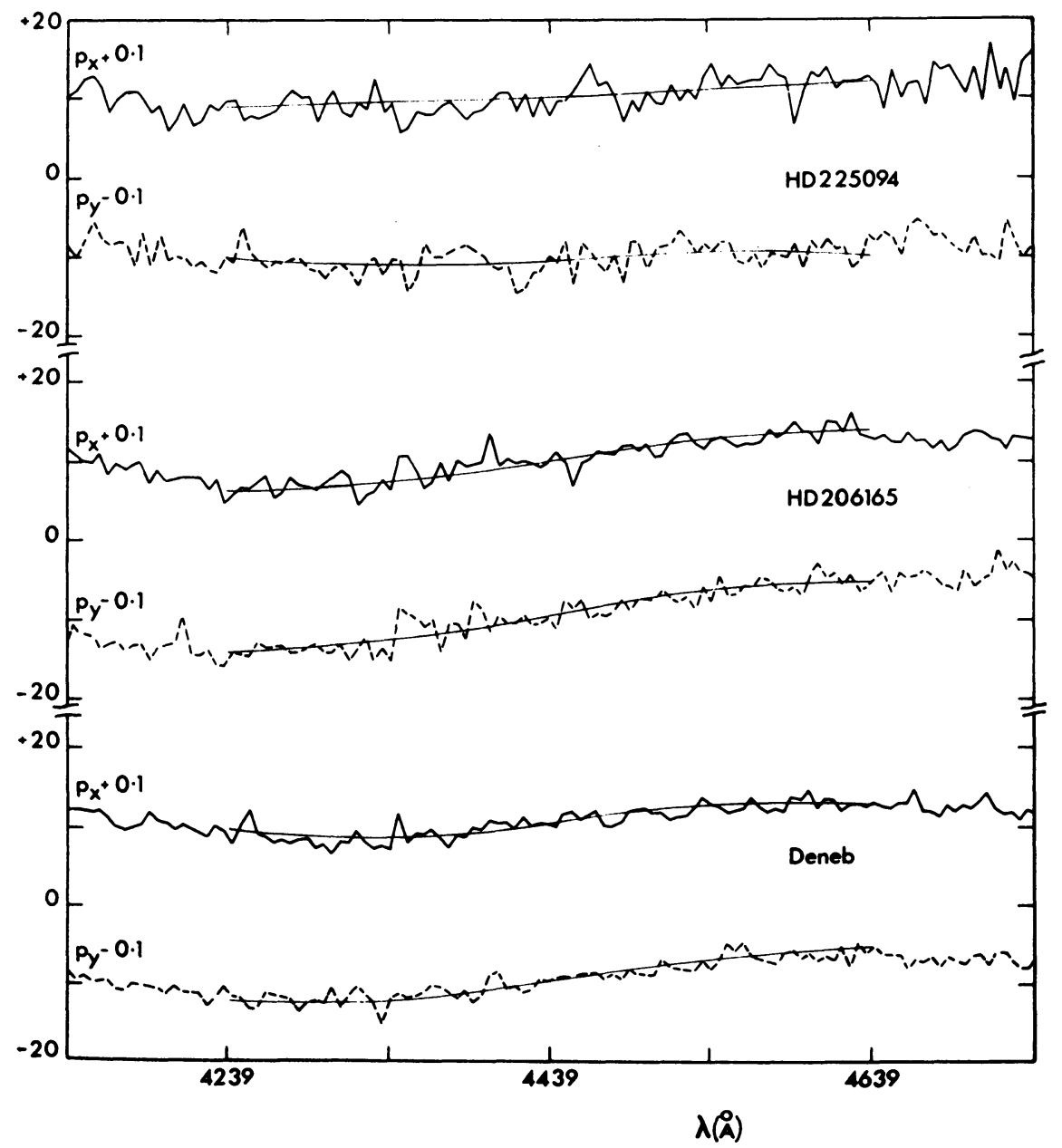

Fig. 2. Wavelength dependance of $p_{x}$ and $p_{y}$ for Deneb ( $\alpha$ Cyg), HD 206165 and HD 225094. 
The noise level of the $\alpha$ Cyg traces is 2.5 times smaller than that of the other two stars, because of the difference in number of spectra used. The 4430 absorption in HD 225094 is much greater than that in HD 206165 and there is indeed an indication of the difference of $p_{x}$ and $p_{y}$ values inside and outside the bands.

We now intend to extend these observations to other stars with large 4430 absorption in different galactic longitudes. We shall then combine these observations appropriately to give weighted mean profiles in these longitudes. We have already obtained spectra of good quality for several stars by using the Wollaston prism at the Cassegrain focus of the $98^{\prime \prime}$ Isaac Newton Telescope and their reduction is in progress.

\section{References}

Brück, M. T. and Nandy, K.: 1968, Nature 220, 46.

Bromage, G.: 1972, Astrophys. Space Sci. in press.

Greenberg, J. M. and Stoeckly, R.: 1970, in L. Houziaux and H. E. Butler (eds.), 'Ultraviolet Stellar Spectra and Related Ground-Based Observations', IAU Symp. 36, 36.

Greenberg, J. M. and Stoeckly, R.: 1971, Nature Phys. Sci. 230, 15.

Hall, J. S. and Serkowski, K.: 1963, Stars and Stellar Systems 3, 293.

Kelly, A.: 1971, Astrophys. Space Sci. 13, 211.

Walker, G. A. H., Hutchings, J. B., and Younger, P. F.: 1970, in L. Houziaux and H. E. Butler (eds.),

'Ultraviolet Stellar Spectra and Related Ground-Based Observations', IAU Symp. 36, 52.

Wickramasinghe, N. C. and Nandy, K.: 1971, Nature Phys. Sci. 229, 234.

York, D. G.: 1971, Astrophys. J. 166, 65. 